Article

\title{
Airside Performance of H-Type Finned Tube Banks with Surface Modifications
}

\author{
Pradhyumn Bhale ${ }^{1} \mathbb{D}$, Mrinal Kaushik ${ }^{1} \mathbb{D}$, Jane-Sunn Liaw ${ }^{2}$ and Chi-Chuan Wang ${ }^{3, * \mathbb{D}}$ \\ 1 Department of Aerospace Engineering, Indian Institute of Technology, Kharagpur 721302, India; \\ pradhyumnbhale12@gmail.com (P.B.); mkaushik@aero.iitkgp.ac.in (M.K.) \\ 2 Green Energy \& Environment Research Laboratories, Industrial Technology Research Institute, Zhutung 310, \\ Taiwan; jsliaw@itri.org.tw \\ 3 Department of Mechanical Engineering, National Chiao Tung University, Hsinchu 300, Taiwan \\ * Correspondence: ccwang@mail.nctu.edu.tw; Tel.: +886-3-571-2121 (ext. 55105)
}

Received: 3 January 2019; Accepted: 3 February 2019; Published: 13 February 2019

check for updates

\begin{abstract}
The present study numerically investigates some novel modifications to augment the performance of the H-type finned tube banks, which are widely used in waste heat recovery in industries. The imposed modifications upon the original H-type finned tube banks include the following: (1) Design 1 contains some triangular cuts at the edge of the original rectangular fin; (2) Design 2 modifies the original rectangular geometry into a trapezoid shape; (3) Design 3 renders the original rectangular cross-section fin thickness into trapezoid cross-section; and (4) Design 4 changes the original rectangular shape into a circular shape. Based on the simulations, it is found that Design 1 shows barely any improvements in the heat transfer performance and surface area reduction. Design 2 can provide some weight saving and surface area reduction at a slightly inferior heat transfer performance. Design 3 can offer up to $14 \%$ improvements in the overall heat transfer performance without any pumping power penalty. Yet, Design 4 provides the maximum weight saving as compared to the original reference case. With 3-9\% lesser surface area than the reference case, Design 4 still offers marginally higher heat transfer performance.
\end{abstract}

Keywords: H-type fin; airside performance; heat transfer augmentation

\section{Introduction}

Finned-tube heat exchangers are widely used for heat exchange between air and liquids. Normally, the thermal resistance on the airside is dominant in the overall heat transfer process. Hence, more fin surface is often needed to ease the gigantic thermal resistance on the airside. Moreover, the problems in using air-cooled heat exchangers may even face a tougher situation in many power plants due to erosion (wear) and fouling by flue gas at the fin and tube surface. One of the surfaces to tailor this problem is the H-type finned tube banks, which provide anti-wear and anti-fouling features due to the presence of unique groove-type structures on their fin surfaces. Yet, the surfaces also possess certain self-cleaning properties due to their special geometrical structure. In essence, this makes H-type finned tube banks one of the best candidates for offering appropriate, reliable, and relatively safe use in severe industry environments while still maintaining a high heat transfer capacity.

In recent years, considerable efforts have been conducted to better understand and improve the airside heat transfer characteristics of H-type finned tube banks. Many experimental and numerical studies on H-type finned tube banks focused on the fin layout in the heat exchangers and the effect of varying geometric parameters like fin width, fin height, fin pitch, fin thickness, and air velocity on the overall heat transfer performance. Furthermore, many geometrical modifications of the fin surface had 
been proposed in the past to improve the heat transfer characteristics of the H-type finned tube banks. A brief overview of some of the past works is listed in the following.

Chen et al. [1] experimentally studied the heat transfer and pressure drop characteristics of H-type finned tube banks with varying geometrical parameters such as fin width, fin height, and fin pitch and provided some useful correlations for the fin efficiency, Nusselt number, and Euler number with respect to air velocity, fin height, fin width, and fin pitch. Yu et al. [2] performed experimental tests on singleand double-H-type finned tube banks and provided some basic correlations on the airside and heat transfer characteristics of H-type finned tube banks. Zhang et al. [3] studied the heat transfer and flow resistance characteristics using a realizable $\mathrm{k}-\varepsilon$ turbulent model based on the FLUENT platform. They showed that the heat transfer coefficient of the H-type finned tube bank increases when raising the air velocity, transverse, and longitudinal pitch of tube banks and with a decrease in fin height and fin pitch. Jin et al. [4] also performed three-dimensional numerical studies using FLUENT. They used the field synergy principle to investigate the geometric influences on the thermofluids' performance, including the effects of the number of tube rows, fin thickness, slit width, fin height, fin pitch, transverse tube pitch, and longitudinal tube pitch subject to the Reynolds number, and their results showed that the transverse tube pitch plays the most important role in the thermofluids' characteristics.

Apart from varying basic geometrical characteristics like fin pitch, fin height, fin width, and fin thickness along with varying the air velocity, many passive techniques were also employed to increase the heat transfer characteristics of H-type finned tube banks. Some researchers adopted vortex generators and dimples to reduce fouling and to enhance the overall heat transfer performance. Wu et al. [5] performed experimental studies concerning the airside performance of two novel fin-tube heat exchangers with delta winglet pairs. It was found that the heat transfer was enhanced with a reduction of pressure loss. Zhao et al. [6] conducted experimental research on the heat transfer and pressure drop of the H-type finned tube bank with longitudinal vortex generators (LVGs) and dimples. It was found that the H-type finned tube bank with dimple-LVG improved the energy recovery and improved the anti-fouling effects by decreasing the ash accumulation on the tube surface. Zhao et al. [7] performed numerical simulations on the heat transfer and erosion characteristics for the H-type finned oval tube with longitudinal vortex generators and dimples. It was found that LVGs and dimples not only improve the heat transfer performance, but also reduce the wear loss of heat exchangers. Many papers also investigated the performance augmentation via using an oval tube instead of the more traditional circular base tube [6,7]. In essence, the H-type finned oval tube bank can reduce the wear loss and penetration attack of the tube surface in comparison with the traditional H-type finned circular tube.

For further improvement upon plate-fin and tube heat exchanger, there have been modifications done by many researchers. For example, Chen and Wang [8] investigated different fin patterns in rectangular, trapezoidal, and an inverted trapezoidal configuration, reporting $10 \%$ reduction in thermal resistances for specified pumping power. Kraus et al. [9] summarized detailed studies of rectangular, triangular, trapezoidal, concave parabolic, and convex parabolic profiles of extended fin surfaces. Despite that these designs may offer some considerable improvements, none of the aforementioned studies were associated with H-type finned tube banks that were popularly used in chemical processing and petroleum industry. Normally, the H-type finned tube bank houses a huge volume and weight that is not only costly, but also bulky as far as installation is concerned. In this regard, it is the objective of this study to elaborate some possible ways to reduce the surface area (or weight) of the H-type finned bank while maintaining the associated heat transfer performance. Both "partial-bypass", trapezoidal, and wedge configurations have been proposed, and it will be shown subsequently that a maximum $14 \%$ improvement can be achieved. The reference case (denoted as Bank X) and a schematic of the typical H-type finned bank are given in Figure 1. 

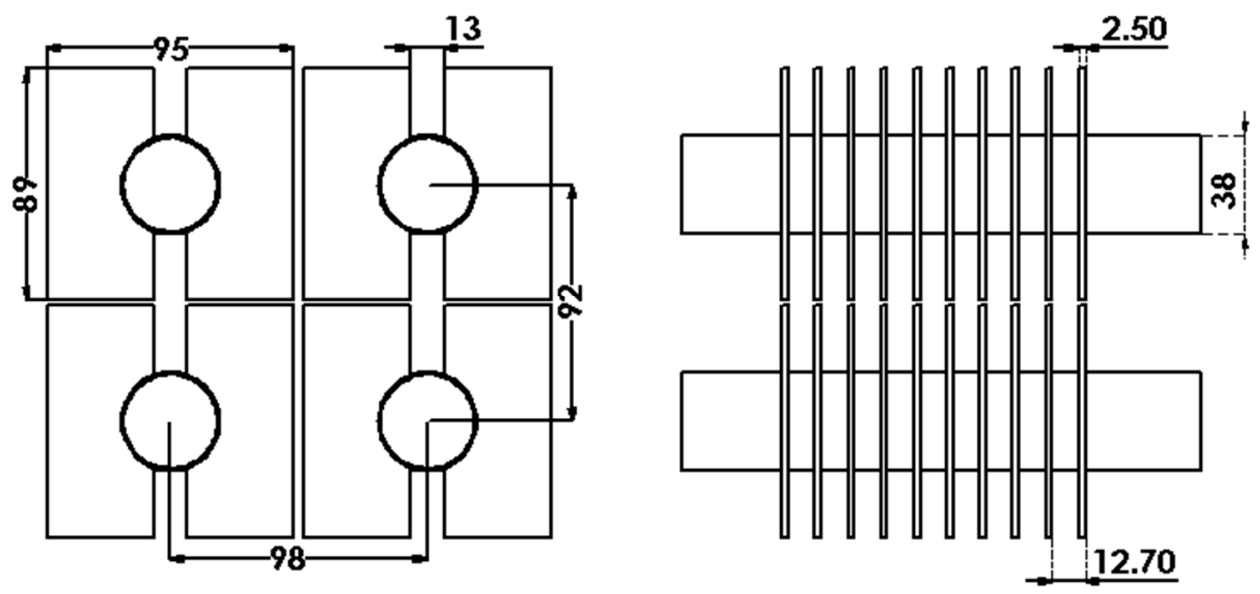

Figure 1. A schematic diagram of a typical H-type finned tube bank with rectangular cross-section (Bank X) and with all dimensions labelled in $\mathrm{mm}$.

\section{Model Description and Numerical Method}

\subsection{Physical Model}

\subsubsection{Design 1}

The modifications in Design 1 are shown in Figure 2a with a1 denoting the cutting length of the upper part from the air inlet, a2 the upper cut at the inner side (tube facing side), $b$ the lower cut at the outer side, and $c$ the upper cut in the bottom array of fins of the outer side. Different combinations of the simulations have been tabulated in Table 1 (the original $\mathrm{H}$-shaped tube bank without any cuts is termed as Bank X). The modifications are based on the idea of "partial bypass" by Wang et al. and Chen and Wang $[10,11]$. Since the heat transfer performance at the entrance of the heat sink (heat exchanger) often far exceeds that of the rear part of the heat sink, as a consequence, one can introduce some bypass airflow that is not in close contact with the surface at the entrance. Some part of the air then flows toward the rear part of the heat sink. Apparently, it will offer a larger temperature difference at the trailing part of the heat sink and a higher heat transfer rate accordingly. Through this manipulation, the heat transfer in the front part may be impaired, but the rear part may be significantly improved. There might exist a niche region by which the performance of the heat sink can be improved.

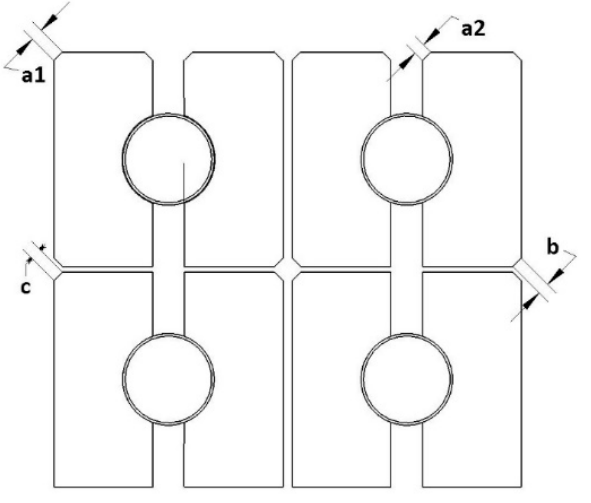

(a) Design 1

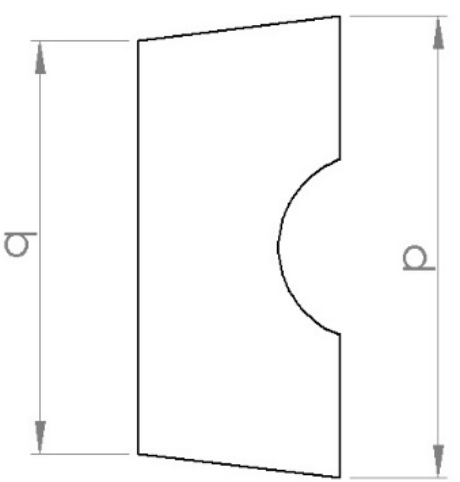

(b) Design 2

Figure 2. Cont. 


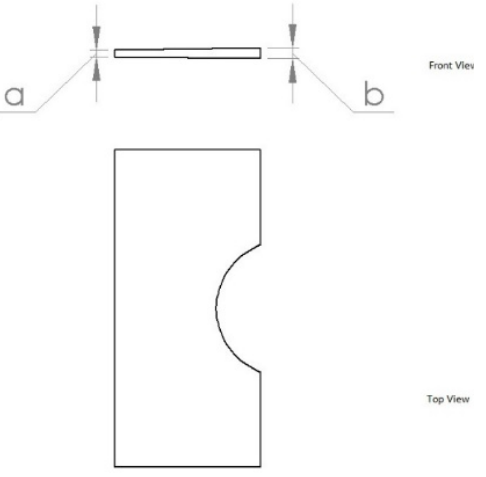

(c) Design 3
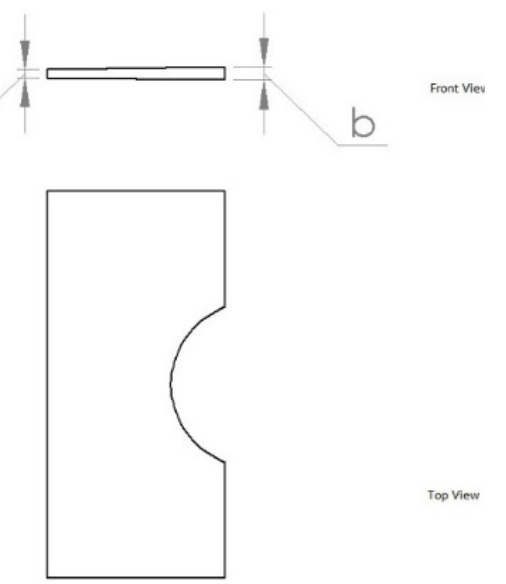

(d) Design 4

Figure 2. Schematic and terminology dimension for Designs 1 4.

Table 1. Nomenclature of the characteristic dimension for the different designs used in the present simulation.

\begin{tabular}{|c|c|c|c|c|c|c|}
\hline \multirow[t]{2}{*}{ Design } & \multirow[t]{2}{*}{ Bank Name } & \multicolumn{4}{|c|}{ Characteristic Dimension for Various Designs } & \multirow{2}{*}{$\begin{array}{c}\text { Weight Reduction (\%) } \\
\text { Relative To Reference Bank X }\end{array}$} \\
\hline & & a1 (mm) & a2 (mm) & b (mm) & $\mathrm{c}(\mathrm{mm})$ & \\
\hline \multirow{6}{*}{ Design 1} & B1 & 4.24 & - & - & - & 0.09 \\
\hline & B2 & 4.24 & - & - & 4.24 & 0.18 \\
\hline & $\mathrm{C} 1$ & 5.2 & - & - & - & 0.13 \\
\hline & $\mathrm{C} 2$ & 5.2 & - & - & 5.2 & 0.26 \\
\hline & $\mathrm{C} 3$ & 5.2 & - & 5.2 & 5.2 & 0.39 \\
\hline & E1 & 7.07 & - & - & - & 0.22 \\
\hline Reference & $X$ & \multicolumn{2}{|c|}{89} & \multicolumn{2}{|c|}{89} & \\
\hline \multirow{2}{*}{ Design 2} & $\mathrm{~K}$ & \multicolumn{2}{|c|}{89} & \multicolumn{2}{|c|}{84} & 2.96 \\
\hline & $\mathrm{L}$ & \multicolumn{2}{|c|}{94} & \multicolumn{2}{|c|}{79} & 2.92 \\
\hline & Bank Name & a (mm) & b (mm) & Ratio (b:a) $[\beta]$ & Cross-Section Type & \\
\hline Reference & $X$ & 2.5 & 2.5 & $1: 1$ & Rectangular & \\
\hline Design 3 & $\mathrm{P}$ & 2 & 3 & $3: 2$ & Wedge-shaped & 1.46 \\
\hline Design 4 & $\mathrm{Z}$ & & & 51.65 & & 3.79 \\
\hline
\end{tabular}

\subsubsection{Design 2}

The modification is schematically shown in Figure $2 b$ with characteristic dimensions $p$ and $q$. The configuration contains a trapezoid fin shape alongside the flow direction. The fin surface takes the 
form as a trapezoid from the side view of the H-type heat exchanger while maintaining a uniform fin thickness. A couple of values for $p$ and $q$ are examined with the nomenclature for the banks as tabulated in Table 1, and the results for heat transfer and material saving are discussed later in subsequent sections. For the reference case of Bank $\mathrm{X}$, ' $\mathrm{p}$ ' and ' $\mathrm{q}$ ' are equal to $89 \mathrm{~mm}$.

\subsubsection{Design 3}

In this design, the tube banks have a varying fin thickness while keeping the fin height and the fin width the same as the reference Bank X. The ratio of the fin tip to the fin base thickness is denoted as $\beta$. A typical diagram for Design 3 is shown in Figure 2c. Tube banks with different values of $\beta$ have been tabulated in Table 1 with the tube bank having a rectangular cross-section $(\beta=1)$ becoming the reference Bank X. Note that the simulated cases for Design 3 with different $\beta$ all contain the same volume (no weight change in fins).

\subsubsection{Design 4}

In this design, semi-circular fins are used instead of the generally-used rectangular fins for the H-type finned tube banks. A typical diagram for this tube bank is shown in Figure $2 \mathrm{~d}$. The improvement in heat transfer performance and material saving for this design are discussed subsequently. Table 1 also shows the value of the outer radius of the fins, and the rest of the parameters like fin thickness, gap between subsequent layers, number of tube rows, fin pitch, and base tube diameter are kept the same as that of the original Bank X.

\subsection{Governing Equations and Turbulence Solver}

The gas-solid flow in the test section is assumed three-dimensional, viscous, and steady incompressible turbulent flow. The realizable k- $\varepsilon$ model with enhanced wall treatment is used to model the turbulent flow, and the corresponding equations are listed below:

Continuity equation:

$$
\frac{\partial}{\partial x_{i}}\left(\rho u_{i}\right)=0
$$

Momentum equation:

$$
\frac{\partial}{\partial x_{i}}\left(\rho u_{i} u_{j}\right)=\frac{\partial}{\partial x_{i}}\left(\mu \frac{\partial u_{j}}{\partial x_{i}}\right)-\frac{\partial p}{\partial x_{i}}
$$

Energy equation:

$$
\frac{\partial}{\partial x_{i}}\left(\rho u_{i} u_{j}\right)=\frac{\partial}{\partial x_{i}}\left(\mu \frac{\partial u_{j}}{\partial x_{i}}\right)-\frac{\partial p}{\partial x_{i}}
$$

The modeled transport equations for $\mathrm{k}$ and $\varepsilon$ in the realizable $\mathrm{k}-\varepsilon$ model are:

$$
\begin{gathered}
\frac{\partial(\rho \kappa)}{\partial t}+\frac{\partial\left(\rho \kappa u_{j}\right)}{\partial x_{j}}=\frac{\partial}{\partial x_{j}}\left(\left[\mu+\frac{\mu_{t}}{\sigma_{\kappa}}\right] \frac{\partial \kappa}{\partial x_{j}}\right)+G_{\kappa}+G_{b}-\rho \epsilon-Y_{M}+S_{\kappa} \\
\frac{\partial(\rho \epsilon)}{\partial t}+\frac{\partial\left(\rho \epsilon u_{j}\right)}{\partial x_{j}}=\frac{\partial}{\partial x_{j}}\left(\left[\mu+\frac{\mu t}{\sigma \epsilon}\right] \frac{\partial \epsilon}{\partial x_{j}}\right)+\rho C_{1} S_{\epsilon}-\rho C_{2} \frac{\epsilon^{2}}{\kappa+\sqrt{v \epsilon}}+C_{1_{\epsilon}} \frac{\epsilon}{\kappa} C_{3_{\epsilon}} G_{b} S_{\epsilon}
\end{gathered}
$$

where $C_{1}=\max \left[0.43, \frac{\eta}{\eta+5}\right], \eta=S \frac{k}{\epsilon}, S=\sqrt{2 S_{i j} S_{i j}}$,

In these equations, $G_{K}$ represents the generation of turbulence kinetic energy due to the mean velocity gradients. $G_{b}$ is the generation of turbulence kinetic energy due to buoyancy, and $Y_{M}$ represents the contribution of the fluctuating dilatation in compressible turbulence to the overall dissipation rate. $C_{2}$ and $C_{1} \varepsilon$ are constants. $\sigma_{\kappa}$ and $\sigma_{\varepsilon}$ are the turbulent Prandtl numbers for $\kappa$ and $\epsilon$, respectively. $S_{K}$ and $S_{\varepsilon}$ are user-defined source terms. 
The standard k- $\epsilon$ model provides more accurate results for considerably high Reynolds number flows. ANSYS ${ }^{\mathrm{R}}$ Fluent provides three types of $\mathrm{k}-\epsilon$ models to be used for turbulence modeling, namely standard k- $\epsilon$, the Re-Normalization Group (RNG) k- $\epsilon$ model, and the realizable k- $\epsilon$ model. Though generally, $k-w$ shear stress transport (SST) formulation are used for simulating low Reynolds number flows, many researchers use $k-\epsilon$ models with different wall treatments as this requires less computation time and resources. Further, it is also found that the RNG k- $\epsilon$ model and realizable k- $\epsilon$ model provide accurate results in numerical simulations (Zhao et al. [7]). They found that the RNG $\mathrm{k}-\epsilon$ model aligned nicely with their experimental data. The main drawback with $\mathrm{k}-\epsilon$ model is that it is not very accurate in the near-wall computations. ANSYS ${ }^{R}$ Fluent has thus provided options for overcoming this shortcoming in these models by having an option of "Enhanced Wall Treatment" in the Near-Wall Treatment option with further selections of the pressure gradient effects and thermal effects in the near-wall region along with viscous heating. If the near-wall mesh is fine enough to address the laminar sublayer, then this method is quite similar to how the solver treats the turbulent modeling in the near-wall region in the k-w SST model. Through this manipulation, contact sizings of the fins and the base tube region were added so that the mesh refinement is done carefully. The advantage of using this wall-enhanced treatment for the k- $\epsilon$ model is that the resultant model has lower computational requirements, which is considered to be advantageous in a number of practical engineering applications. A comparison of the results in terms of thermal resistance versus pumping power using the realizable $k-\epsilon$ model with enhanced wall treatment and the $k-w$ SST model is shown in Figure 3. The results reveal an insignificant difference in the results obtained by using these two aforesaid models, and hence, the numerical solutions for all the designs considered in this study are obtained using the realizable k- $\epsilon$ model with enhanced wall treatment.

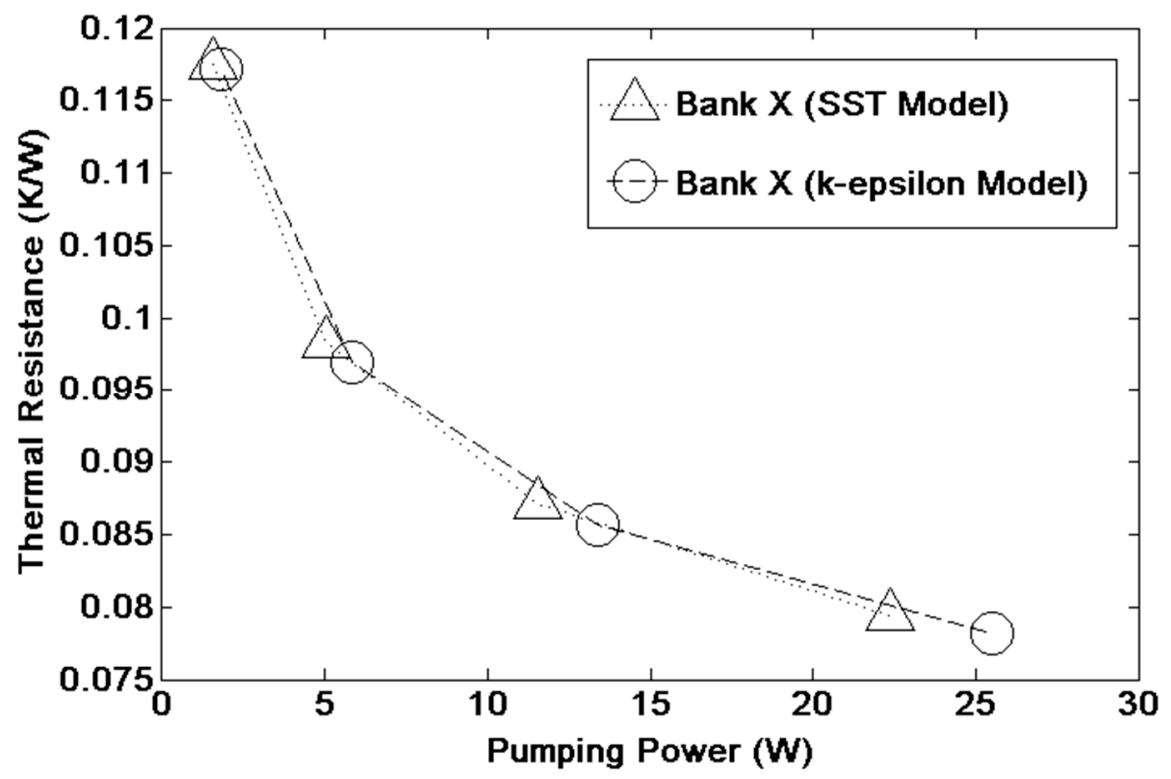

Figure 3. Comparison of results for Bank X using realizable k- $\epsilon$ and k-w SST models.

\subsection{Boundary Conditions and Thermo-Physical Properties}

At the upstream inlet, the fluid entering the computational domain is assumed to have uniform velocity and turbulent intensity with velocity components in the $y$ - and z-directions being set to zero. Considering the symmetry of the test setup, a symmetric boundary condition is used along the flow direction so as to reduce the computational time for each test case. At the symmetry planes, the normal velocity component, the heat flux, and the normal first derivatives of other variables are also set to be zero. Periodic boundary conditions are used at the opposite ends, which are indeed perpendicular to the flow inlet direction (i.e., along the spanwise direction) to extend the flow domain. 
The base tube is maintained at a constant temperature of $\mathrm{T}_{\mathrm{W}}\left(\right.$ or $\mathrm{T}_{\text {cold }}$ ) $=308 \mathrm{~K}$, and the wall thickness of the tube surface is ignored. The hot air temperature at the inlet is $376 \mathrm{~K}\left(\mathrm{~T}_{\text {hot }}\right)$. The cell zone conditions for the tube are also maintained at a constant temperature. The difference in the results that are obtained, by using the constant temperature boundary condition and by the convective heat transfer boundary condition, for the tube wall surface are negligibly small.

The material used for both the fins and tube surface is carbon steel $(1.5 \% \mathrm{C})$. The working fluid is air and all properties are evaluated at normal temperature and pressure. Since the thermo-physical properties of air like specific heat, thermal conductivity, dynamic viscosity, etc., change rather little with temperature range in the present simulations, thereby the influence of changes in the properties is neglected. All the banks are simulated with air inlet velocities ranging from $6-15 \mathrm{~m} / \mathrm{s}$. When the residual of each variable for gas phase is below $10^{-4}$, the numerical simulation is regarded as convergence.

\subsection{Meshing}

The mesh is created in ANSYS Workbench using the inbuilt meshing feature in the Fluid Flow (Fluent) solver. The mesh for Bank X contains 3,458,898 elements with around 600,000 nodes. The advanced sizing functions are used over both proximity and curvature with high smoothing and slow transition. Eighty contact sizings of $2 \mathrm{~mm}$ each for the fin and base tube-contact regions are also used. The mesh is more structured over the fin surfaces and unstructured over the contact regions. The average orthogonal quality for the mesh elements is 0.89 with a standard deviation of less than 0.09 . The typical mesh for the fin and tube surfaces for Bank X with a zoom in is shown in Figure 4a. The meshing for all the other designs is carried out using similar settings, as well.

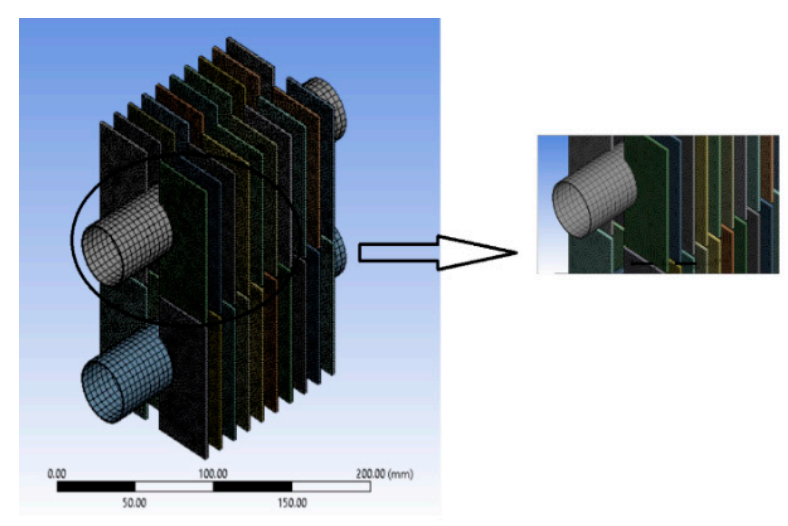

(a) Meshing schematic

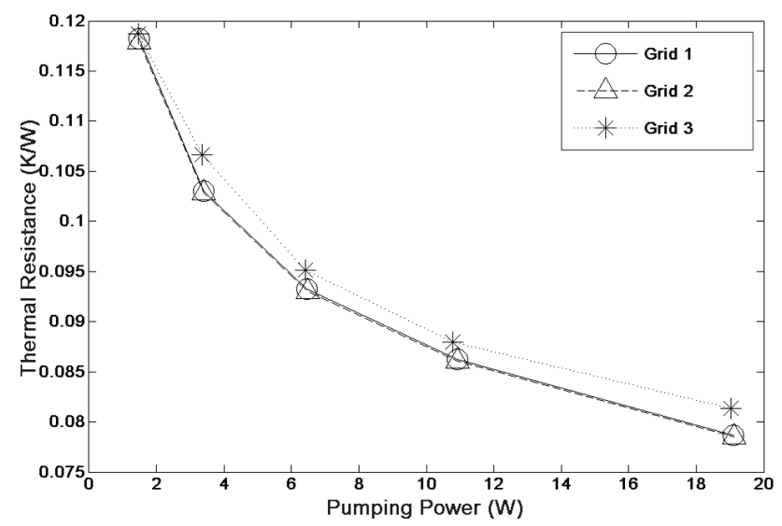

(b) Grid independence test

Figure 4. Meshing schematic and grid independence test for the three grids. 


\subsection{Grid Independence Test and Validation of the Computational Model}

In order to validate the grid independency of the solutions, three-different mesh systems are investigated for the original H-type finned tube bank without any cuts (Bank X) using 2,670,583 (Grid 1), 3,458,898 (Grid 2), and 5,270,254 (Grid 3) cells. The comparison of the results for the three grids are shown in Figure $4 \mathrm{~b}$ in a graph in terms of thermal resistance vs. pumping power. Apart from the grid-independency test, the numerical results were compared with the experimental data from Chen et al. [1]. The results for the heat transfer coefficient $(\mathrm{h})$ and pressure drop $(\Delta \mathrm{P})$ for mesh with $3,458,898$ elements are plotted against the experimental values in in Figure 5a,b. The simulations are in line with the experimental results with $6 \%$ difference in predictive heat transfer coefficient $(\mathrm{h})$ and the measurements, while being within $20 \%$ for the pressure drop $(\Delta \mathrm{P})$. The verification of the present simulation against the experimental results substantiates the applicability of the present simulations.

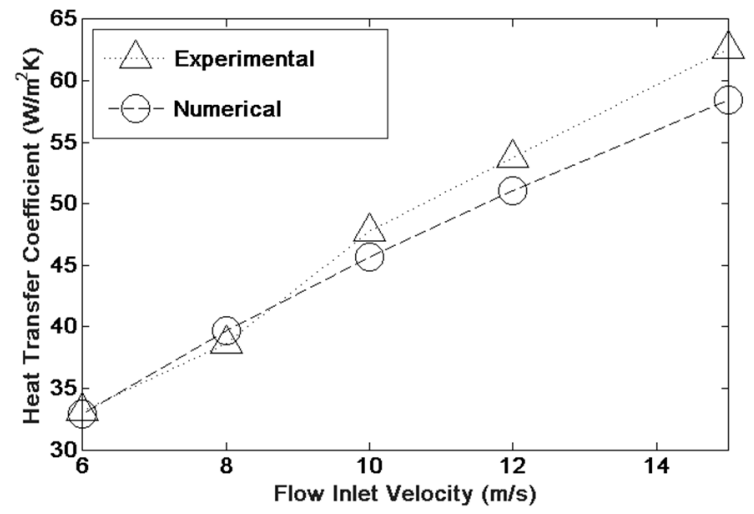

(a)

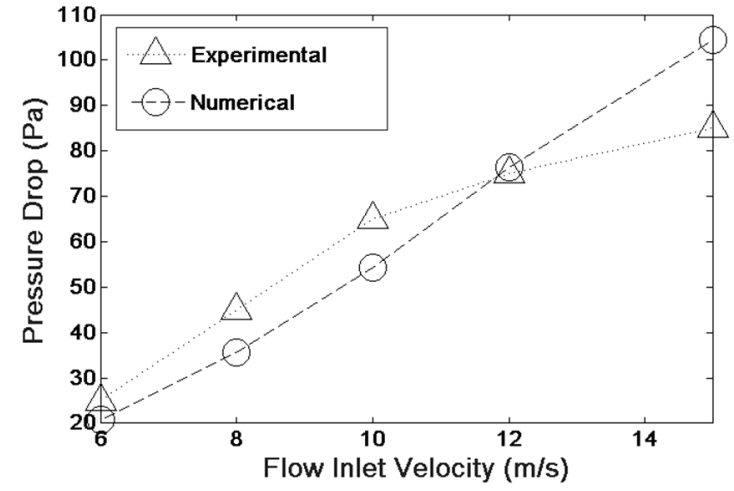

(b)

Figure 5. Comparison of experimental (Chen et al. [1]) and numerical results for: (a) heat transfer coefficient; (b) pressure drop.

\section{Results and Discussion}

In the following sections, the typical temperature contours for various tube banks are described, and the fin efficiency is defined and discussed for the tube banks. The thermal resistance against varying pumping powers is compared with different designs of finned tube banks as described earlier. Surface area reduction for material saving along with the possible improvement in heat transfer performance is the prime objective of this study.

\subsection{Temperature Contours and Fin Efficiency}

A typical temperature contour for Bank X at an inlet velocity of $12 \mathrm{~m} / \mathrm{s}$ is shown in Figure 6a. A close observation of the temperature contours indicates that the fin portions that are farther away from the base tube have a higher value of average temperature. These high temperature portions thereby impose an adverse influence on the overall efficiency of the tube banks and deteriorate the heat transfer performance accordingly. The fin efficiency, $\eta$, is defined as the ratio of the actual heat transfer and the given heat transfer assuming that the fin surface temperature is equal to that of the base tube. It is expressed as:

$$
\eta_{f}=\frac{\oiiint h\left(T-T_{\infty}\right) d F_{f}}{h\left(T_{\text {cold }}-T_{\infty}\right) F_{f}}
$$

where $T_{\infty}$ is the hot air inlet temperature, $T_{\text {cold }}$ is the base tube temperature, and $F_{f}$ is the fin surface area. Considering uniform heat transfer coefficient across the fin surface, the fin efficiency can be approximated to:

$$
\eta_{\mathrm{f}}=\frac{\frac{\oiint \mathrm{T}_{\mathrm{f}} \mathrm{dF}_{\mathrm{f}}}{\mathrm{F}_{\mathrm{f}}}-\mathrm{T}_{\text {hot }}}{\left(\mathrm{T}_{\text {cold }}-\mathrm{T}_{\text {hot }}\right)}
$$


Hence,

$$
\eta_{\mathrm{f}}=\frac{\mathrm{T}_{\text {hot }}-\mathrm{T}_{\text {avg }}}{\mathrm{T}_{\text {hot }}-\mathrm{T}_{\text {cold }}}
$$

where $\mathrm{T}_{\text {hot }}$ is the hot inlet temperature ( $\left.376 \mathrm{~K}\right), \mathrm{T}_{\text {cold }}$ is the base tube temperature ( $\left.308 \mathrm{~K}\right)$, and $\mathrm{T}_{\text {avg }}$ is the average surface temperature of the entire heat transfer surface, which includes the exposed fin and base tube surfaces. Using these ideas, fins with side cuts, trapezoidal fins, wedge-shaped cross-sections, and circular fins are used for subsequent numerical simulations. The tube banks with considerable fin thickness near the base tube can provide substantial improvement in the heat transfer performance and improve the overall efficiency due to reduction in the average temperature of the fin surface. The improvements in the overall heat transfer performance can range from $2 \%-14 \%$ for the studied designs; while the circular fins show the maximum saving in material's usage with around $10 \%$ reduction in the material cost and deliver approximately the same heat transfer performance. In fact, an additional $2 \%$ reduction in thermal resistance can be achieved by nearly $4 \%$ reduction in material usage. Typical temperature contours for different design are shown in Figure $6 \mathrm{~b}-\mathrm{d}$.
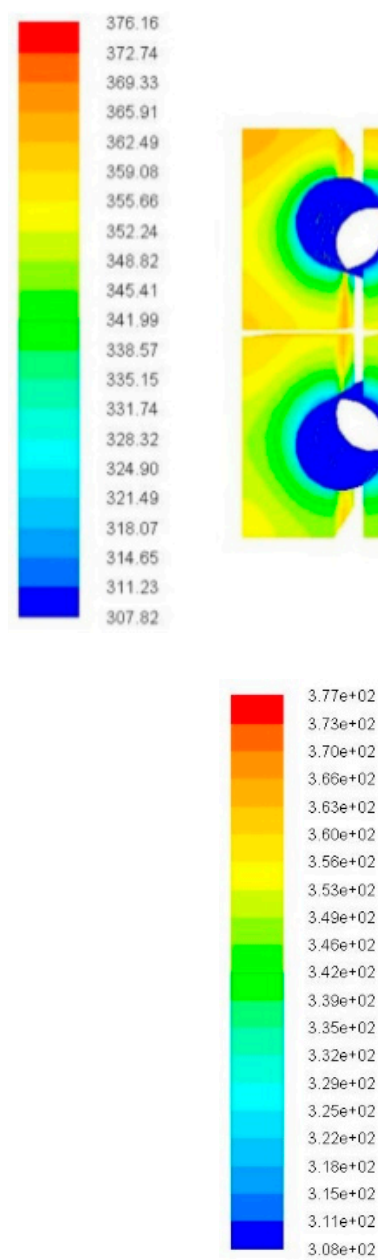
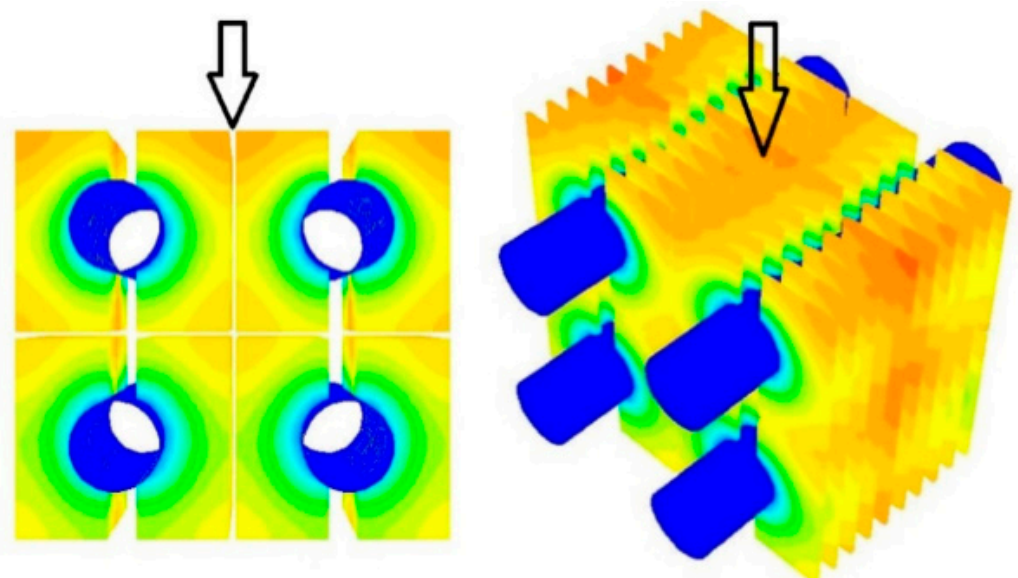

(a)

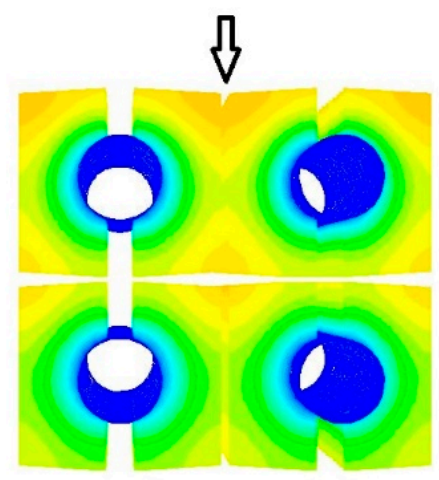

(b)

Figure 6. Cont. 


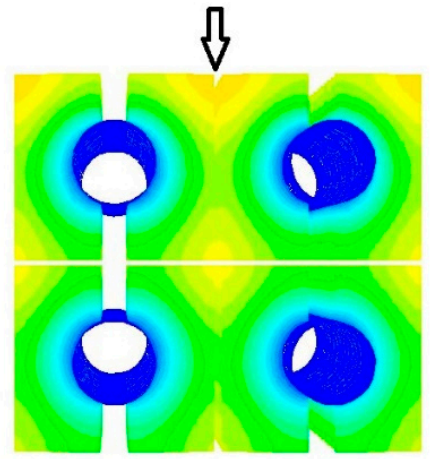

(c)
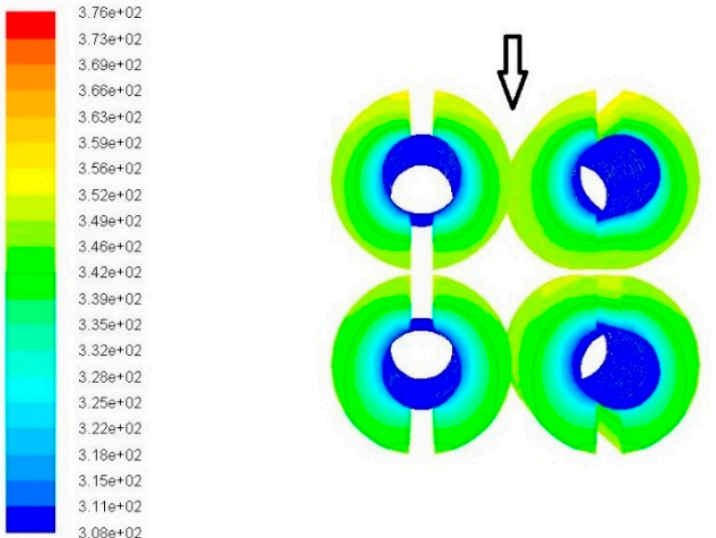

(d)

Figure 6. Typical temperature contours for the designs: (a) Bank X; (b) Bank 1 in Design 2; (c) Bank P in Design 3; (d) Bank Y in Design 4.

\subsection{Thermal Resistance vs. Pumping Power for Different Tube Banks}

The thermal resistances and pumping power for the different tube banks as a measure with that of Bank $X$ are shown in Figure 7. The thermal resistance and pumping power is evaluated as:

The pumping power is calculated as follows:

$$
\mathrm{W}=\dot{\forall} \Delta \mathrm{P}
$$

where $\mathrm{W}$ is pumping power, $\dot{\forall}$ is volumetric flow rate, and $\Delta \mathrm{P}$ is pressure drop across the tube bank. The average thermal resistance stands for the overall heat transfer characteristics of the heat sink design, which was calculated as follow:

$$
\mathrm{R}_{\mathrm{a}}=\frac{\mathrm{T}_{\mathrm{w}}-\mathrm{T}_{\mathrm{a}}}{\mathrm{Q}}, \mathrm{T}_{\mathrm{a}}=\frac{\left(\mathrm{T}_{\text {out }}+\mathrm{T}_{\text {in }}\right)}{2}
$$

where $R_{a}$ is the average thermal resistance, $T_{w}$ is the tube wall temperature, $T_{a}$ is the average temperature of the airflow, and $\mathrm{Q}$ is the heat transfer rate of the tube bank. $\mathrm{T}_{\text {in }}$ and $\mathrm{T}_{\text {out }}$ are the inlet and outlet air temperature, respectively. 


\subsubsection{Design 1}

For Design 1 as depicted in Figure 7a-c, generally, it is comparatively effective to have the cut at the exterior edge (e.g., cut at a1 in Figure 2a), rather than the inner cut (e.g., cut at a2 in Figure 2a). For the typical a1 cut at the exterior region, the thermal resistances are roughly the same for the same pumping power as shown in Figure 7a for Cases B1-B3. By comparing the results, it is easy to see that Bank $\mathrm{C} 1$ provides the best performance improvement as seen in Figure $7 \mathrm{~b}$, where 2-3\% reduction in thermal resistance can be achieved at the same pumping power. Yet, $\mathrm{C} 1$ offers a very tiny weight reduction in material of just about $0.4 \%$. On the other hand, the performance is appreciably degraded by about 7\% at the same pumping power with inner cut (a2) of Bank E4, as shown in Figure 2c. Hence, cutting this area, a2, will jeopardize the overall heat transfer performance and increase the thermal resistance accordingly. It is also interesting to know that the thermal resistance for Bank E4 at the same power, e.g., 10-15 W, is considerably higher than E1. The only difference amid E1 and E4 is the location of the surface cut. The surface cut for E1 is at the exterior entrance of the H-type fin, while it is at the central part of the entrance for E4. There are two reasons for this difference. Firstly, the influence of fin efficiency on overall performance for E4 is more pronounced than that of E1, yet the heat transfer coefficient at this regime is also comparatively larger than that at the exterior entrance region because the a 2 cut is located toward the stagnation direction onto the tube row where the heat transfer coefficient is much higher than the exterior regions. This is because air flows perpendicularly into the H-type edge at this portion, while it is slightly inclined at the exterior entrance. In summary, regarding these influences, it is more effective to place the surface cut at the exterior entrance portion. Design 1 shows negligible improvement in the heat transfer performance subject to cutting. The major benefit of Design 1 is maintaining the performance with very small material saving. However, the maximum material saving is only around $0.07 \% \sim 0.31 \%$, suggesting that cutting alongside the surface either at the first or second row is not so effective after all.

\subsubsection{Design 2}

Design 2 modifies the original design into a trapezoid shape. As shown in Figure 7d, Bank K and Bank L provide marginally higher thermal resistance subject to the same pumping power as that of Bank X. Actually, the slight rise of thermal resistance is attributed to the slight reduction in surface area (about $3 \%$ ) in comparison with the reference Bank X. The results suggest that changing the fin shape into a trapezoid may be less effective.

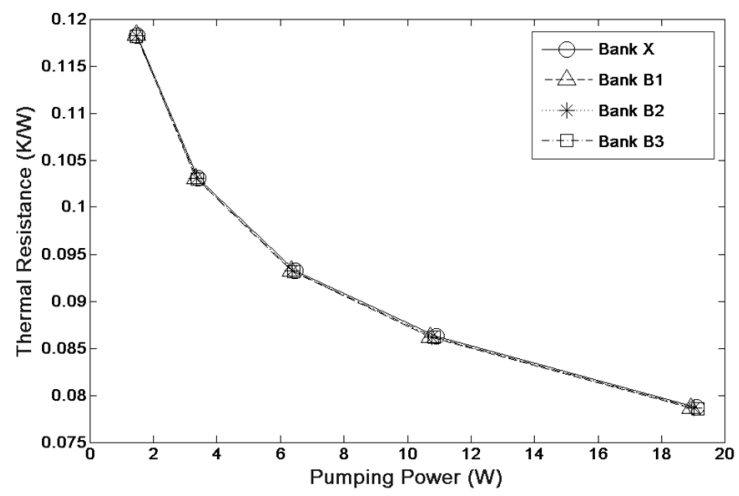

(a)

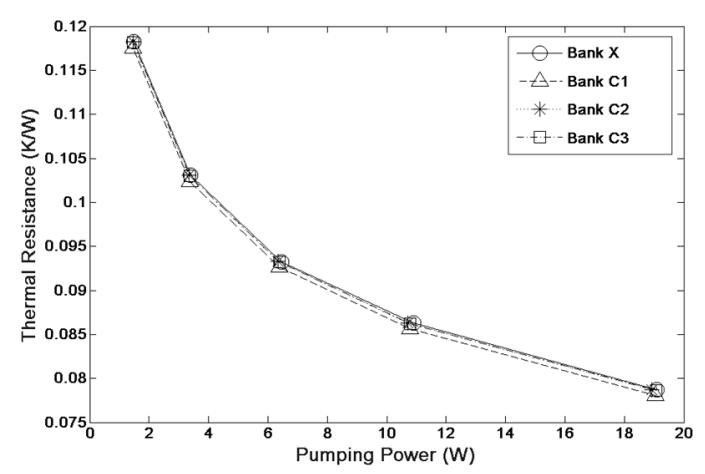

(b)

Figure 7. Cont. 


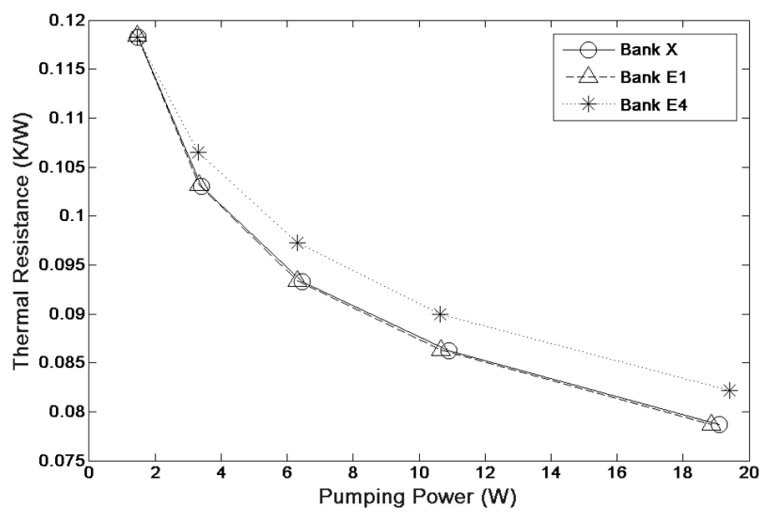

(c)

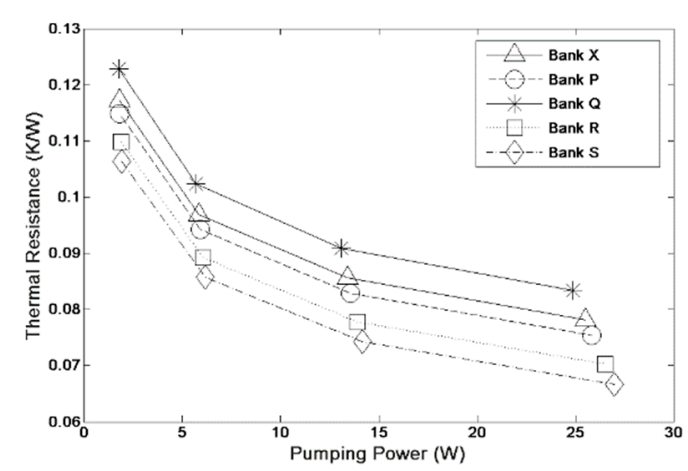

(e)

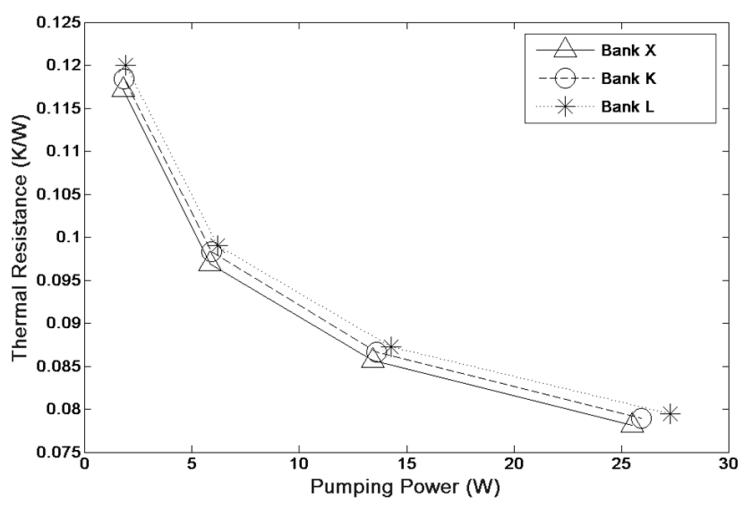

(d)

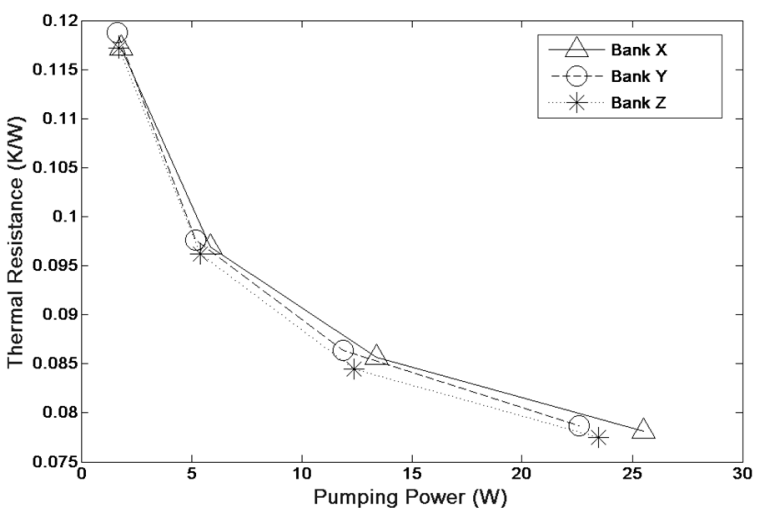

(f)

Figure 7. Thermal resistance vs. pumping power: (a-c) Design 1; (d) Design 2; (e) Design 3; (f) Design 4.

\subsubsection{Design 3}

In Design 3, the modification is made by changing the cross-section surface area of the original H-type fin into a wedge-shaped cross-section while maintaining the rest of the geometric parameters. Unlike Design 2, which contains a trapezoid shape that is parallel to the flow direction, the wedge-shaped cross-section offers a much superior thermal performance than the rest of configurations. This is in line with the analytical prediction for the trapezoid heat sink used for electronic cooling by Chen and Wang [8]. In fact, Design 3 shows the maximum improvement in the overall heat transfer performance. Comparing the results, it is easy to see that as the values of $\beta$ increases ( $\beta$ is defined as $b / a$, the ratio of trapezoid base to trapezoid tip; $\beta=1$ represents the original rectangular profile), the overall heat transfer performance of the tube banks also increases. By varying the original Bank $X$ to Bank $P$, Bank $R$, and Bank $S$, the value of $\beta$ increases from 1.5, 4, and 49 , respectively, and thermal resistance shows a persistent decrease subject to pumping power, while the improvement in thermal resistance reduction for Bank $\mathrm{P}$ is around $2-3.5 \%$, Bank $\mathrm{R}$ from $6-10 \%$, and Bank S from 9-14\%. Tube banks with $\beta<1$ were also tested, and no improvement in the heat transfer performance was observed, while only Bank B with $\beta=0.67$ is shown here. The results are graphically shown in Figure 7e. In summary, regarding Designs 2 and 3, it is comparatively effective to have a fin with a wedge-shaped cross-section rather than a trapezoid fin shape with a uniform thickness. Notice that another benefit of using the wedge type design is the material saving, as tabulated in Table 1, where the Bank $\mathrm{S}$ can offer about 7\% material saving. 


\subsubsection{Design 4}

In this design, circular-shaped fins are used instead of rectangular fins. Bank $\mathrm{Y}$ and Bank $\mathrm{Z}$ are tested. Bank $Z$ shows around a $2 \%$ drop in the thermal resistance, while Bank $Y$ has nearly the same heat transfer performance in comparison with Bank $X$, as shown in Figure $7 f$. Moreover, the effective surface area for circular-shaped fins is actually lower than the reference H-type fin. The surface reduction is about $8 \%$ for Bank Y, while it is $3.76 \%$ for Bank $Z$. Despite containing less surface area, the circular-shaped fins are still marginally better than that of the original fin design. This can be made clear from the surface temperature contour for the original and the circular-shape one, as shown in Figure $6 a, d$, respectively. It appears that the circular fin provides more uniformity in surface temperature distribution, which leads to a comparatively high fin efficiency and a better heat transfer performance accordingly.

\subsection{Area Reduction and Weight Saving}

The weight reduction of the proposed designs as compared to Bank $X$ is also tabulated in the last column of Table 1. With cutting at the outer corners of the fin surfaces in association with the circular fins, the present authors are able to achieve surface area reduction for the heat exchanger, which varies in scale for the different finned tube banks. In Design 1, by raising the size of the cuts, the area reduction is generally small. However, a further increase of the cut size over $5.2 \mathrm{~mm}$ (Bank C1), the thermal performance starts falling due to too much loss in surface area. In Design 2 and Design 4, some area reduction can be achieved for Bank K, Bank Y, and Bank Z, while maintaining a comparable heat transfer performance. Yet, Design 3 has been examined by keeping the weight the same as that of Bank X. Through this design, it can provide a significant improvement in thermal performance without the penalty of additional pumping power. The results imply that the wedge shape is applicable in the finned tube heat exchanger, as well as applications like an electronic cooling heat sink. Notice that Design 4 offers the best surface area reduction, $8 \%$, for Bank $\mathrm{Y}$ without the pumping power penalty.

\subsection{Effect of Increasing the Separation between Fin Rows}

In the original Bank $X$, the separation distance between the tube rows along the longitudinal direction is taken as $3 \mathrm{~mm}$. The gap between the two tube rows can be further increased to $6 \mathrm{~mm}$, $10 \mathrm{~mm}$, and $20 \mathrm{~mm}$, respectively. As shown in Figure 8, the heat transfer performance increases slightly around $1-2 \%$ when the separation distance between tube rows is increased. This is because a longer separate distance can offset the secondary flow effect behind the tube. Additionally, the increase of separation distance offers a side advantage for easier cleaning and reducing fouling, but it also increases the effective volume of the heat exchanger. 


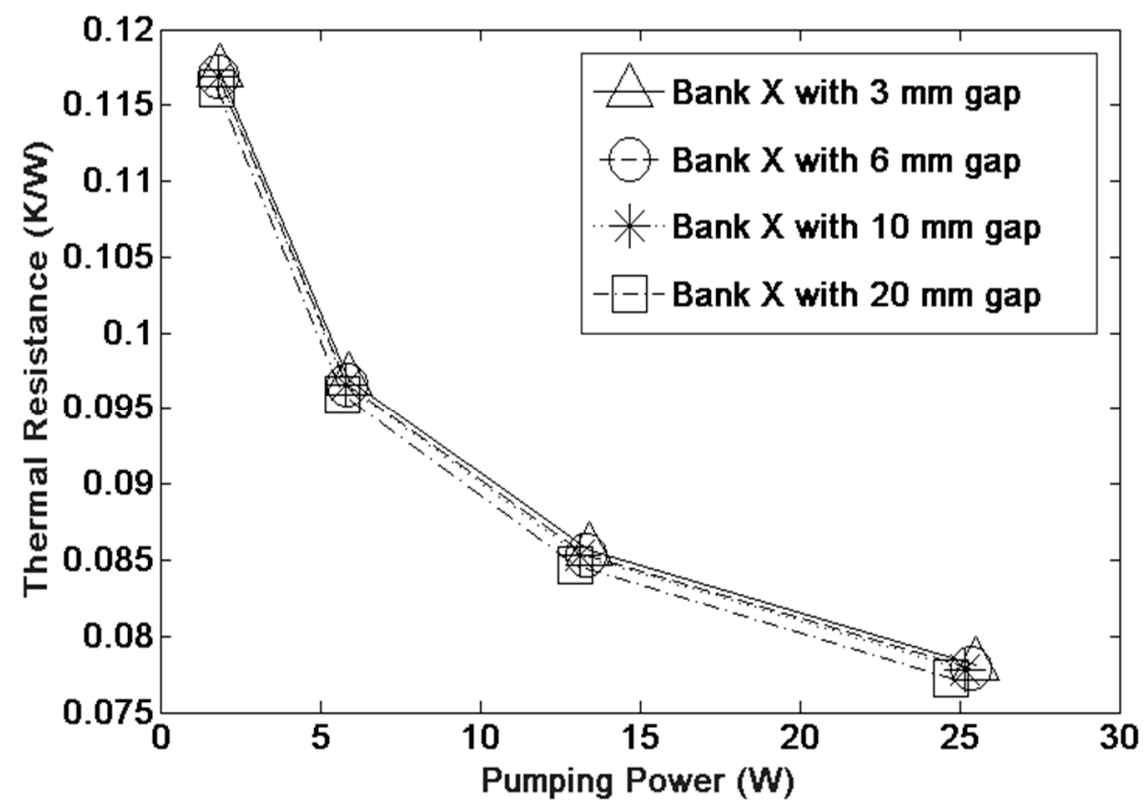

Figure 8. Thermal resistance vs. pumping power at varying gaps between adjacent rows of the finned tube bank.

\section{Conclusions}

In this study, the heat transfer performance of H-type finned tube banks subject to different augmentations has been carried out. The modifications on the original H-type finned tube banks include the following: (1) Design 1 offers some triangular cuts at the edge of the original rectangular fin; (2) Design 2 modifies the original rectangular geometry into a trapezoid shape; (3) Design 3 renders the original rectangular cross-section fin thickness into a trapezoid cross-section; and (4) Design 4 changes the original rectangular shape into a circular shape. The foregoing designs make use of some possible surface/weight reduction and explore the possibility of the heat transfer augmentation. The designs are numerically investigated and compared with the traditional H-type fin surface with a rectangular cross-section, namely Bank X. Based on the foregoing discussion, the following conclusions are made as follows:

1. The fins with triangular cuts towards the edges (in Design 1) show very small improvements in the heat transfer performance and surface area reduction.

2. Bank K and Bank L (Design 2) adopting trapezoid fin shape can provide some weight saving and surface area reduction at a cost of slightly inferior heat transfer performance.

3. The fin surface with a wedge-shaped cross-section (Design 3) can offer up to $14 \%$ improvements in the overall heat transfer performance without any pumping power loss. The fin surface with a reverse wedge-shaped cross-section (thinner towards the base tube) imposes a negative influence on the overall thermal resistance.

4. The circular-shaped fins (Design 4) provide the maximum reduction in the weight as compared to the original reference case. With a 3-9\% reduction in the surface area, the design still offers marginally higher heat transfer performance than the reference case. This is because it can provide a higher fin efficiency.

Author Contributions: All the authors have contributed their efforts to complete the paper. P.B. conducted the numerical calculations and wrote the first draft. M.K. co-wrote the first draft. J.-S.L. supervised the numerical study. C.-C.W. supervised the work and reviewed and edited the manuscript.

Funding: This research was funded by Ministry of Science and Technology, Taiwan, grant number 107-2622-E-009-002-CC2 and 107-2221-E-009-142 and support from the Bureau of Energy, Ministry of Economic Affairs of Taiwan. 
Acknowledgments: The authors are indebted to the financial support from the Bureau of Energy, Ministry of Economic Affairs, Taiwan, and grants from the Ministry of Science and Technology, Taiwan, under Contracts 107-2622-E-009-002-CC2 and 107-2221-E-009-142.

Conflicts of Interest: The authors declare no conflict of interest.

\section{Nomenclature}

a1

a2

b

C

$\mathrm{F}_{\mathrm{f}}$

h

$\mathrm{p}$

q

Q

$\mathrm{R}_{\mathrm{a}}$

$\mathrm{T}_{\text {hot }}$

$\mathrm{T}_{\text {in }}$

$\mathrm{T}_{\mathrm{W}}$ or $\mathrm{T}_{\text {cold }}$

$\mathrm{T}_{\text {avg }}$

$\mathrm{T}_{\text {out }}$

W

$\beta$

$\eta_{\mathrm{f}}$

$\Delta \mathrm{P}$

$\dot{\forall}$

In Design 1, cutting length of the upper part, away from the tube-side of first layer of fins, $\mathrm{m}$

In Design 1, cutting length of the upper part, towards the tube-side of first

layer of fins, $\mathrm{m}$

In Design 1, cutting length of the lower part, away from the tube-side of first layer of fins, $\mathrm{m}$

In Design 1, cutting length of the upper part, away from the tube-side of second layer of fins, $\mathrm{m}$

Fin surface area, $\mathrm{m}^{2}$

Heat transfer coefficient of the heat exchanger, $\mathrm{W} / \mathrm{m}^{2} \mathrm{~K}$

In Design 2, length of fin surface at the tube facing side, $\mathrm{m}$

In Design 2, length of fin surface away from the tube facing side, $\mathrm{m}$

Heat transfer rate, $\mathrm{W}$

Thermal resistance, $\mathrm{K} / \mathrm{W}$

Hot air inlet temperature, $\mathrm{K}$

Inlet air temperature, $\mathrm{K}$

Base tube temperature of the tube bank, $\mathrm{K}$

Average fin surface and exposed base tube temperature, $\mathrm{K}$

Outlet air temperature, $\mathrm{K}$

Pumping power, $\mathrm{W}$

In Design 3, the ratio of thickness at the base tube to the tip of the fin

surface, dimensionless

Fin efficiency of the finned heat exchanger, dimensionless.

Pressure drop across the tube bank, $\mathrm{Pa}$

Volumetric flow rate, $\mathrm{m}^{3} / \mathrm{s}$

\section{References}

1. Chen, H.; Wang, Y.; Zhao, Q.; Ma, H.; Li, Y.; Chen, Z. Experimental Investigation of Heat Transfer and Pressure Drop Characteristics of H-type Finned Tube Banks. Energies 2014, 7, 7094. [CrossRef]

2. Yu, X.N.; Yuan, Y.C.; Ma, Y.F.; Liu, H.L. Experimental tests and numerical simulation on heat transfer and resistance characteristics of H-type finned tube banks. Dongli Gongcheng Xuebao (J. Chin. Soc. Power Eng.) 2010, 30, 433-438.

3. Zhang, Z.; Wang, Y.; Zhao, Q. Numerical Study on Performance Optimization of H-type Finned Tubes. J. Power Eng. 2010, 30, 941-946.

4. Jin, Y.; Tang, G.-H.; He, Y.-L.; Tao, W.-Q. Numerical Study of the Solid Particle Erosion on H-Type Finned Circular/Elliptic Tube Surface. Commun. Comput. Phys. 2017, 21, 466-489. [CrossRef]

5. Wu, J.M.; Zhang, H.; Yan, C.H.; Wang, Y. Experimental study on the performance of a novel fin-tube air heat exchanger with punched longitudinal vortex generator. Energy Convers. Manag. 2012, 57, 42-48. [CrossRef]

6. Zhao, X.; Tang, G.; Shi, Y.; Li, Y. Experimental Study of Heat Transfer and Pressure Drop for H-type Finned Oval Tube with Longitudinal Vortex Generators and Dimples under Flue Gas. Heat Transf. Eng. 2017, 1-9, 608-616. [CrossRef]

7. Zhao, X.B.; Tang, G.H.; Ma, X.W.; Jin, Y.; Tao, W.Q. Numerical investigation of heat transfer and erosion characteristics for H-type finned oval tube with longitudinal vortex generators and dimples. Appl. Energy 2014, 127, 93-104. [CrossRef]

8. Chen, H.-L.; Wang, C.-C. Analytical analysis and experimental verification of trapezoidal fin for assessment of heat sink performance and material saving. Appl. Therm. Eng. 2016, 98, 203-212. [CrossRef]

9. Kraus, A.D.; Aziz, A.; Welty, J. Extended Surface Heat Transfer; John Wiley \& Sons: Hoboken, NJ, USA, 2002. 
10. Wang, C.-C.; Chen, K.-Y.; Liaw, J.-S.; Tseng, C.-Y. A novel "partial bypass" concept to augment the performance of air-cooled heat exchangers. Int. J. Heat Mass Transf. 2012, 55, 5367-5372. [CrossRef]

11. Chen, C.-H.; Wang, C.-C. A novel trapezoid fin pattern applicable for air-cooled heat sink. Heat Mass Transf. 2015, 51, 1631-1637. [CrossRef] 